\title{
Asociación entre la magnitud del viraje del hematocrito y otros indicadores de severidad en dengue
}

\author{
Fredi Alexander Díaz-Quijano, MD, MSc ${ }^{1}$, Ruth Aralí Martínez-Vega, MD, MSc ${ }^{1}$, \\ Luis Ángel Villar-Centeno, MD, MSc ${ }^{2}$
}

\section{RESUMEN}

Objetivos: Establecer la asociación entre la magnitud del viraje del hematocrito y otros hallazgos de severidad en dengue. Diseño: Estudio observacional analítico de corte transversal.

Población y métodos: Pacientes con diagnóstico clínico y serológico (IgM positiva) de dengue, hospitalizados en instituciones de Santander (Colombia), durante 1993-1998. Se evaluó la asociación entre la intensidad de la hemoconcentración y complicaciones como hemorragias espontáneas, efusiones pleurales y trombocitopenia $(<50,000 \mathrm{plt} / \mu \mathrm{l})$.

Resultados: Se incluyeron 780 pacientes. En un análisis multivariado, las variables asociadas con una mayor hemoconcentración fueron: edad $<15$ años, hematemesis y trombocitopenia. Hubo 363 pacientes con hemoconcentración $<10 \%$; 226 entre $10 \%$ y $20 \%$; y 191 con un viraje del hematocrito $>20 \%$. La frecuencia de efusiones pleurales no fue diferente entre estos grupos. La hematemesis y la trombocitopenia fueron menos frecuentes en el grupo con hemoconcentración $<10 \%$. Además, estas complicaciones fueron más frecuentes con hemoconcentración $>20 \%$ vs. 10\%-20\% ( $<<0.05$ ). Comparado con un viraje $>20 \%$, un criterio más flexible de hemoconcentración ( $>10 \%)$ se asoció con un aumento de la sensibilidad de $31 \%$ a $63 \%$ y una disminución de la especificidad de $88.5 \%$ a $66.3 \%$, para detectar alguna complicación (hematemesis, trombocitopenia o efusión pleural).

Conclusión: Este estudio sugiere una fuerte correlación entre la intensidad de la hemoconcentración y la severidad del dengue. Además, sugiere que al emplearse un criterio más flexible para definir la extravasación plasmática, se obtiene un importante incremento en la sensibilidad con una aceptable disminución en la especificidad. Estos resultados deben evaluarse prospectivamente, en un estudio de cohortes.

Palabras clave: Dengue; Severidad; Hematocrito; Extravasación plasmática; Hemorragias espontáneas.

\section{Correlation between variation of hematocrit and other indicators of severity in dengue}

\section{SUMMARY}

Objectives: To determine the association of hemoconcentration with other signs of severity of dengue.

Design: Cross sectional study.

Population and methods: Patients with clinical and serologic diagnosis (IgM positive test) of dengue, who consulted hospitals in Santander (Colombia), during 1993-1998. The clinical findings and laboratory tests were registered. Associations among hemoconcentration and complications, such as spontaneous hemorrhages (petechiae, ecchymoses, bleeding from gums, epistaxis, hematemesis, hematuria and metrorrhagia), pleural effusions and thrombocytopenia $(<50,000 \mathrm{plt} / \mu \mathrm{l})$, were evaluated.

Results: A sampling of 780 patients was included. In a multi-varied analysis, thrombocytopenia $(\mathrm{p}<0.001)$, hematemesis $(\mathrm{p}=0.04)$, and age under 15 years $(\mathrm{p}=0.02)$ were associated to greater hemoconcentration. There were 363 patients with hemoconcentration $<10 \% ; 226$ with $10 \%-20 \%$; and 191 with $>20 \%$. There were no significant differences in frequency of pleural effusions within these groups. Hematemesis and thrombocytopenia were less frequent in patients with hemoconcentration $<10 \%$ compared with both, $10 \%-20 \%$ and $>20 \%$ groups. Moreover, patients with hemoconcentration $>20 \%$ had more

1. Investigador, Grupo de Epidemiología Clínica, Centro de Investigaciones Epidemiológicas, Universidad Industrial de Santander(CIE-UIS), Bucaramanga,Colombia.e-mail:fre_diazq@yahoo.com rutharam@yahoo.com

2. Profesor Titular, Escuela de Medicina, Universidad Industrial de Santander, Bucaramanga, Colombia. e-mail: luisangelvillarc@yahoo.com

Recibido para publicación mayo 14, 2008 Aceptado para publicación septiembre 30, 2009 
complications than the group with hemoconcentration of $10 \%-20 \%(\mathrm{p}<0.05)$. Compared with $>20 \%$, a hematocrit change $>10 \%$ was associated to increasing sensitivity from $31 \%$ to $63.4 \%$ and diminishing of specificity from $88.5 \%$ to $66.3 \%$, to detect some complications (hematemesis, thrombocytopenia, or pleural effusion).

Conclusions: This study suggests a strong correlation between hemoconcentration and severity of dengue. Also, more liberal criteria to define plasma leakage could be used. The present results should be prospectively evaluated in cohort studies.

Keywords: Dengue; Severity; Hematocrit; Plasma leakage; Spontaneous hemorrhages.

El dengue es la enfermedad generada por arbovirus, más importante en el mundo ${ }^{1}$. La infección aguda se puede presentar como un síndrome febril agudo acompañado de cefalea, dolor ocular, mialgias, artralgias, exantema y leucopenia ${ }^{2}$. Algunos pacientes desarrollan además, hemorragias espontáneas, trombocitopenia y signos de extravasación plasmática. La conjunción de estas manifestaciones define el síndrome de dengue hemorrágico (DH), que se asocia con una mayor letalidad ${ }^{3}$.

La variación del hematocrito se ha considerado un parámetro importante para clasificar la severidad de la enfermedad. Hasta ahora, la OMS ha considerado que una hemoconcentración mayor de $20 \%$ es un hallazgo que documenta la extravasación de plasma ${ }^{3}$. Sin embargo, aun en pacientes hospitalizados, es difícil documentar este criterio de hemoconcentración, debido a que muy pocos casos tienen este cambio en el hematocrito ${ }^{4-}$ ${ }^{7}$. Por lo anterior, algunos autores han propuesto y usado un criterio de extravasación plasmática más flexible, por ejemplo una hemoconcentración $>10 \%$, a fin de mejorar la sensibilidad de la definición de $\mathrm{DH}^{8,9}$.

Por otra parte, pocos estudios respaldan la asociación el hematocrito y la severidad del dengue ${ }^{8-13}$. En cambio, los trabajos disponibles se han orientado hacia la identificación de un valor único de hematocrito como indicador de severidad, sin validar el viraje mismo como un criterio de complicación ${ }^{8-13}$. En consecuencia, el objetivo del presente artículo es determinar la asociación entre la magnitud del cambio del hematocrito y complicaciones del dengue, como las efusiones pleurales, las hemorragias y la trombocitopenia y evaluar el punto de corte de $10 \%$ en la variación del hematocrito como criterio de severidad.

\section{MATERIALES Y MÉTODOS}

Se realizó un estudio observacional analítico de corte transversal, con pacientes hospitalizados en instituciones de salud de Santander (Colombia), durante el período de 1993 a 1998, con evidencia de infección aguda por el virus del dengue.

Criterios deinclusión. Síndrome febril agudo acompañado de dos o más de las siguientes manifestaciones: cefalea, mialgias, dolor ocular, artralgias, exantema y leucopenia; y una prueba de anticuerpos IgM antidengue positiva (especificidad: $99.1 \%$; valor predictivo positivo en Bucaramanga, Santander: $96.2 \%)^{14}$. Además, los enfermos evaluados debían contar con información clínica y de laboratorio suficiente para clasificar la enfermedad, e incluir por lo menos dos hematocritos tomados en días distintos.

Encuesta. La información se obtuvo retrospectivamente a partir del registro clínico-epidemiológico que se diligenció en el momento de tomar la muestra para el diagnóstico serológico. En el registro se incluyen los síntomas, signos y los valores de hematocrito y plaquetas, determinados en la atención inicial y a diario, durante el período de hospitalización. Por el método de ELISA de captura se hizo la medición de anticuerpos IgM específicos contra dengue, en muestras de suero obtenidas después del quinto día de enfermedad.

Según la Resolución Nº 8430 de 1993 del Ministerio de Salud (Artículo 16), este estudio corresponde a una investigación sin riesgo debido al origen y el carácter de los datos, por lo que no requirió una firma de consentimiento informado.

Desenlaces. Se evaluó la presencia de manifestaciones hemorrágicas (prueba de torniquete, petequias, equimosis, gingivorragia, epistaxis, hematemesis, hematuria y metrorragia), signos clínicos de extravasación plasmática (efusión pleural, edema de miembros inferiores) y trombocitopenia profunda $(<50,000 \mathrm{plt} / \mu \mathrm{l})$.

Almacenamiento de la información y análisis estadístico. Los datos demográficos, clínicos y serológicos, se almacenaron en una base de datos electrónica (Microsoft Excel 97, Microsoft Corp). Las definiciones de los desenlaces se aplicaron de modo sistemático a todos los pacientes.

Para calcular la magnitud de la hemoconcentración, de cada persona se tomó la diferencia entre el hematocrito 


\section{Cuadro 1}

Cambio del hematocrito asociado con el sexo, la edad y el tiempo de evolución de los síntomas al ingreso, en pacientes con dengue

\begin{tabular}{|c|c|c|c|}
\hline Característica & $\mathbf{n}$ & Viraje del hematocrito (IC95\%) & $\mathbf{p}$ \\
\hline \multicolumn{4}{|l|}{ Sexo } \\
\hline Masculino & 436 & $14.8(12.7-16.9)$ & 0.18 \\
\hline Femenino & 344 & $16.9(14.6-19.2)$ & \\
\hline \multicolumn{4}{|l|}{ Edad } \\
\hline$\geq 15$ años & 564 & $14.7(13.1-16.3)$ & 0.04 \\
\hline$<15$ años & 216 & $18.3(14.6-22.1)$ & \\
\hline \multicolumn{4}{|l|}{ Tiempo de enfermedad } \\
\hline$>4$ días & 431 & $17.6(15.1-20.2)$ & 0.006 \\
\hline$\leq 4$ días & 349 & $13.3(11.8-14.8)$ & \\
\hline
\end{tabular}

máximo y el mínimo y se dividió por este último. Este viraje del hematocrito se expresó como porcentaje.

Al principio, con la prueba $t$ de Student, se calculó la asociación entre los signos de severidad y el porcentaje de hemoconcentración. Luego, las asociaciones encontradas se evaluaron en un análisis de regresión linear, en el que se incluyeron las variables de sexo, edad $(<15$ años), tiempo de evolución de los síntomas al ingreso $(>4$ días); y las otras condiciones que mostraban alguna relación con el cambio en el hematocrito $(\mathrm{p}<0.2)$.

También se evaluó la asociación entre las principales complicaciones (hematemesis, derrame pleural y trombocitopenia profunda) y la proporción de viraje del hematocrito, distribuido éste en los rangos de: $<10 \%, 10$ $20 \mathrm{y}>20 \%$. Por último, se determinó la sensibilidad y la especificidad de los puntos de corte de $10 \%$ y de $20 \%$, para descubrir tales complicaciones. En el análisis de datos se empleó el programa estadístico STATA, versión 9.2

\section{RESULTADOS}

Se obtuvo información de 1,119 individuos, de ellos a 904 se les realizó una prueba de IgM específica para dengue, que fue positiva en 891 casos. De estos últimos, en sólo 780 hubo información clínica suficiente para determinar el viraje del hematocrito (grupo analizado). El sexo no se relacionó con los cambios en este parámetro, sin embargo, los menores de 15 años y los captados después del cuarto día de enfermedad, mostraron un mayor viraje en la prueba (Cuadro 1).
En el análisis bivariado, la trombocitopenia profunda y las manifestaciones hemorrágicas, como equimosis, hematemesis, melenas, hematuria y metrorragia, se asociaron con una mayor variación del hematocrito (Cuadro 2). El derrame pleural (Cuadro 2) y el edema en los miembros inferiores (datos que no se muestran), no se relacionaron con la magnitud de la hemoconcentración.

En el análisis multivariado, la edad menor de 15 años, la hematemesis y la trombocitopenia profunda, se asociaban consistentemente con una mayor variación del hematocrito. Estas asociaciones no guardaban relación con el sexo, el tiempo de evolución de la enfermedad y otras manifestaciones hemorrágicas (Cuadro 3).

En general, la población estudiada mostró un descenso progresivo del hematocrito tras los días segundo y tercero de evaluación (Gráfica 1). Los individuos con hematemesis, evidenciaron un hematocrito significativamente menor en la tercera medición, al compararlos con los que no tenían esta forma de sangrado $(p<0.001)$. Por otra parte, los que mostraban una trombocitopenia profunda, presentaron un hematocrito significativamente mayor en su primera y segunda evaluación ( $\mathrm{p}<0.001$, en ambos momentos), con un posterior descenso hasta alcanzar valores similares a los del resto de la población (Gráfica 1).

Entotal,363pacientesmostraronun viraje del hematocrito en el rango menor a 10\%, 226 entre $10 \%$ y $20 \%$ y 191 una hemoconcentración mayor a 20\%. En estos grupos no hubo diferencia estadísticamente significativa en la frecuencia de derrame pleural (Cuadro 4). No obstante, la 
Cuadro 2

Complicaciones asociadas con el viraje del hematocrito. Análisis bivariado

\begin{tabular}{|c|c|c|c|c|}
\hline & & $\mathbf{n}$ & Viraje del hematocrito (IC95\%) & $\mathbf{p}$ \\
\hline \multicolumn{2}{|c|}{ Sangrado espontáneo (total) } & 621 & $16.6(14.7-18.5)$ & 0.03 \\
\hline & no & 158 & $12.2(9.9-14.4)$ & \\
\hline \multirow[t]{2}{*}{ Equimosis } & sí & 139 & $20.4(14.7-26.1)$ & 0.005 \\
\hline & no & 640 & $14.7(13.3-16.1)$ & \\
\hline \multirow[t]{2}{*}{ Hematemesis } & sí & 93 & $24.8(18.9-30.7)$ & $<0.001$ \\
\hline & no & 685 & $14.5(12.9-16.1)$ & \\
\hline \multirow[t]{2}{*}{ Melena } & sí & 79 & $23.1(15.2-31)$ & 0.002 \\
\hline & no & 700 & $14.9(13.3-16.4)$ & \\
\hline \multirow[t]{2}{*}{ Gingivorragia } & sí & 152 & $15.5(11.8-19.1)$ & 0.88 \\
\hline & no & 627 & $15.8(14-17.5)$ & \\
\hline \multirow[t]{2}{*}{ Epistaxsis } & sí & 163 & $18.6(14.6-22.6)$ & 0.06 \\
\hline & no & 615 & $15(13.3-16.6)$ & \\
\hline \multirow[t]{2}{*}{ Hematuria } & sí & 54 & $19.4(10.5-28.3)$ & 0.02 \\
\hline & no & 570 & $13.5(12.1-14.8)$ & \\
\hline \multirow[t]{2}{*}{ Metrorragia } & sí & 49 & $22.6(12.5-32.6)$ & 0.05 \\
\hline & no & 295 & $15.9(13.8-18.1)$ & \\
\hline \multirow[t]{2}{*}{ Efusión pleural } & sí & 29 & $17.8(12-23.7)$ & 0.64 \\
\hline & no & 681 & $15.8(14.1-17.6)$ & \\
\hline \multirow[t]{2}{*}{ Trombocitopenia } & sí & 495 & $17.8(15.9-19.7)$ & $<0.001$ \\
\hline & no & 285 & $12(9.3-14.7)$ & \\
\hline
\end{tabular}

Cuadro 3

Condiciones asociadas con un mayor viraje del hematocrito en pacientes con dengue. Análisis multivariado*

\begin{tabular}{lccr}
\hline Condición & Diferencia de la hemoconcentración (\%) & IC 95\% & p \\
\hline Edad $<15$ años & 1.8 & $0.3-3.4$ & 0.02 \\
Hematemesis & 2.5 & $0.1-5.0$ & 0.04 \\
Trombocitopenia profunda & 5.4 & $3.9-6.8$ & $<0.001$ \\
\hline
\end{tabular}

* Este cuadro resume al análisis de regresión lineal. En este modelo se incluyeron las variables de género, edad ( $<15$ años), tiempo de enfermedad ( $>4$ días) y las manifestaciones hemorrágicas relacionadas con el viraje del hematocrito $(p<0.2)$.

hematemesis y la trombocitopenia profunda se asociaron con los rangos de hemoconcentración de $10 \%-20 \%$ y $>20 \%$, comparados con el grupo de pacientes con viraje del hematocrito $<10 \%$. Asimismo, los pacientes con hemoconcentración $>20 \%$ tuvieron más complicaciones que el grupo de 10\%-20\% (Cuadro 4).
Un viraje del hematocrito mayor de $10 \%$ comparado con un valor superior a $20 \%$, se asoció con un incremento de la sensibilidad y con disminución de la especificidad, para descubrir complicaciones como trombocitopenia, efusión pleural o hematemesis (Cuadro 5). 


\section{Cuadro 4}

Complicaciones del dengue de acuerdo con la proporción de viraje del hematocrito

\begin{tabular}{|c|c|c|c|c|c|c|c|c|c|}
\hline \multirow[t]{2}{*}{ Complicación } & \multicolumn{3}{|c|}{$\begin{array}{c}\text { Grupos según la } \\
\text { hemoconcentración }\end{array}$} & \multicolumn{2}{|c|}{$10-20 \%$ vs. $<10 \%$} & \multicolumn{2}{|c|}{$>20 \%$ vs. $<10 \%$} & \multicolumn{2}{|c|}{$>20 \%$ vs. $10 \%-20 \%$} \\
\hline & $\begin{array}{l}<10 \% \\
(n=363)\end{array}$ & $\begin{array}{l}10-20 \\
(n=226)\end{array}$ & $\begin{array}{l}>20 \% \\
(n=191)\end{array}$ & OR (IC 95\%) & $p$ & OR (IC 95\%) & $p$ & OR (IC 95\%) & $p$ \\
\hline \multicolumn{10}{|l|}{ Efusión pleural } \\
\hline $\mathrm{N}^{\circ}(\%)$ & $9(2)$ & $12(5)$ & $8(4)$ & $2.3(0.95-5.3)$ & 0.07 & $1.7(0.7-4.4)$ & 0.26 & $0.8(0.3-1.9)$ & 0.59 \\
\hline Hematemesis & $26(7)$ & $28(12)$ & $39(20)$ & $1.8(1-3.2)$ & 0.03 & $3.3(1.9-5.6)$ & $<0.001$ & $11.8(1.1-3.1)$ & 0.03 \\
\hline $\begin{array}{l}\text { Trombocitopenia } \\
\text { profunda* }\end{array}$ & $183(50)$ & $160(71)$ & $152(80)$ & $2.4(1.7-3.4)$ & $<0.001$ & $3.8(2.6-5.8)$ & $<0.001$ & $1.6(1-2.5)$ & 0.04 \\
\hline $\begin{array}{l}\text { Cualquier } \\
\text { complicación }\end{array}$ & $190(52)$ & $168(74)$ & $161(84)$ & $2.6(1.8-3.8)$ & $<0.001$ & $4.9(3.2-7.6)$ & $<0.001$ & $11.85(1.1-3)$ & 0.01 \\
\hline
\end{tabular}

* Definida como un recuento $\leq 50,000$ plaquetas $/ \mu \mathrm{l}$

Cuadro 5

Evaluación operativa de los puntos de corte para definir hemoconcentración en pacientes con dengue

\begin{tabular}{cccccc}
\hline $\begin{array}{c}\text { Punto de corte para } \\
\text { hemoconcentración }\end{array}$ & $\begin{array}{c}\mathbf{N}^{\circ} \text { casos detectados } \\
\text { (total = 519/780) }\end{array}$ & Sensibilidad & IC 95\% & Especificidad & IC 95\% \\
\hline$>10 \%$ & 329 & 63.4 & $(59.2-67.5)$ & 66.3 & $(60.5-72.1)$ \\
$>20 \%$ & 161 & 31.0 & $(27-35)$ & 88.5 & $(84.6-92.4)$ \\
\hline
\end{tabular}

* Se consideró caso cuando un paciente presentaba cualquiera de las siguientes complicaciones: efusión pleural, hematemesis o trombocitopenia con $\leq 50,000$ plaquetas $/ \mu \mathrm{l}$

\section{DISCUSIÓN}

Lahemoconcentración es un hallazgo de laboratorio característico del dengue. Unvalor alto del hematocrito suele ser la primera anormalidad originada por la extravasación plasmática en el $\mathrm{DH}^{12,13}$. Sin embargo, en este estudio no hubo asociaciones entre los signos clínicos de fuga plasmática y la intensidad de la hemoconcentración. Esto se podría explicar por el reducido número de eventos (efusiones pleurales) notificados en esta población (29/710), que representan un poder pobre para evaluar este desenlace en particular.

La magnitud de la variación del hematocrito se asoció con manifestaciones hemorrágicas, especialmente con la hematemesis, que se puede catalogar como una hemorragia mayor. La pérdida de sangre por esta vía, parece que se refleja en una paulatina disminu- ción del hematocrito en cada nueva valoración (Gráfica 1). De esta forma, el sangrado podría ser un mecanismo importante en la variación del hematocrito en algunos enfermos, al disminuir notablemente el valor del mismo en la convalecencia.

La trombocitopenia profunda se considera un criterio de hospitalización en dengue ${ }^{3}$. La asociación del recuento de plaquetas $<50,000 / \mu 1$ con un mayor viraje del hematocrito, implica otra relación entre la hemoconcentración y la severidad de esta enfermedad. Esta asociación fue independiente de las hemorragias espontáneas y al parecer se basa en mecanismos muy distintos, pues los sujetos con bajos recuentos de plaquetas presentaron hematocritos significativamente más altos en las primeras evaluaciones (Gráfica 1). A manera de hipótesis, la intensidad de la trombocitopenia podría reflejar la magnitud de los mecanismos 
Hematocrito de acuerdo con la presencia de hematemesis

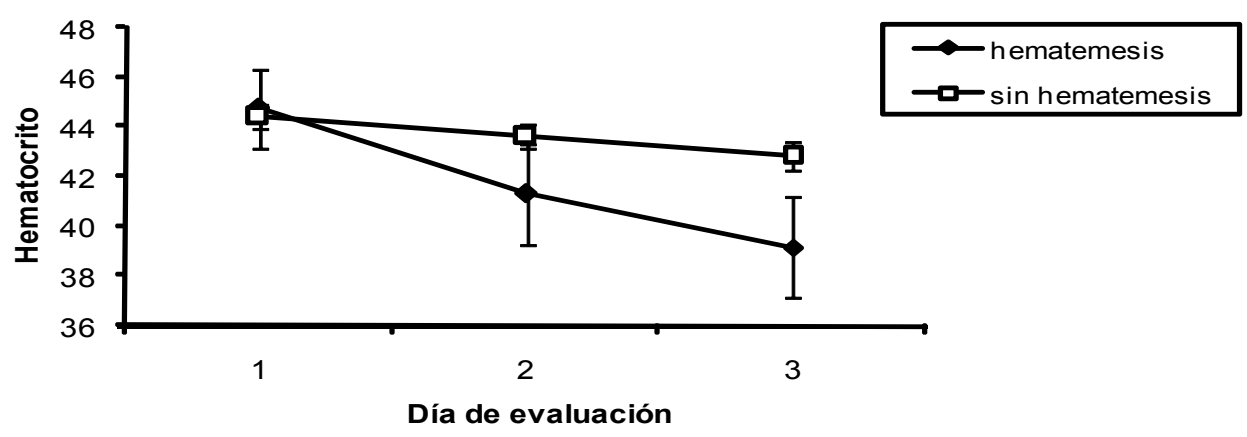

Hematocrito según la intensidad de la trombocitopenia

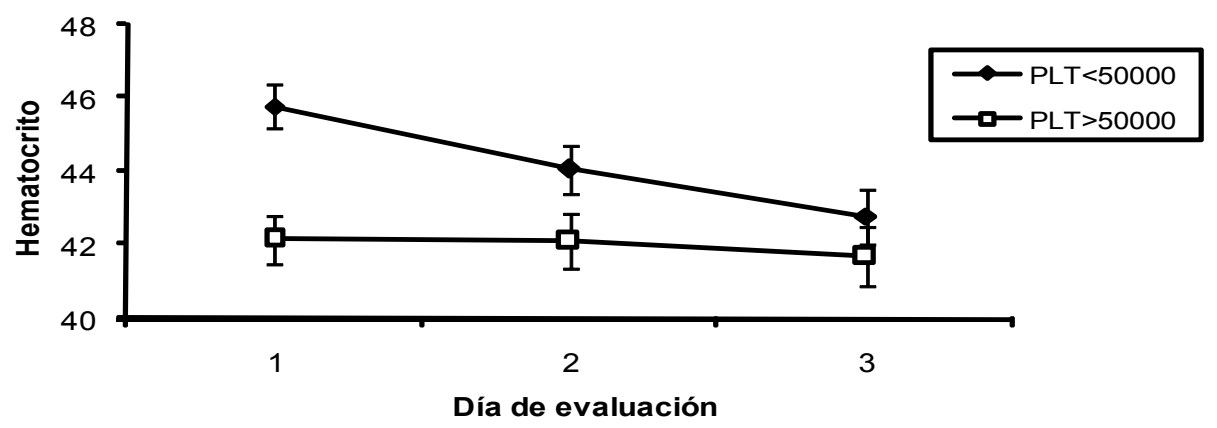

Gráfica 1. Comportamiento del hematocrito según la presencia de hematemesis (arriba) y trombocitopenia profunda (abajo). Las barras de error representan los intervalos de confianza de $\mathbf{9 5 \%}$

inflamatorios de la fase aguda de la enfermedad, que también se asocian con la extravasación plasmática ${ }^{15}$.

Los signos de severidad evaluados fueron significativamente menos frecuentes cuando el viraje del hematocrito estuvo por debajo de $10 \%$. Comparados con éste, los grupos con los rangos de 10\%-20\% y $>20 \%$, exhiben un incremento significativo de complicaciones. Aunque el grupo con viraje $>20 \%$ tiene más complicaciones que aquel con viraje de $10 \%-20 \%$, la magnitud de esta diferencia es mucho menor que la observada al comparar cualquiera de estos dos grupos con aquel de viraje $<10 \%$ (Cuadro 4 ).

Como criterio de severidad, la hemoconcentración mayor de $10 \%$ comparada con un punto de corte de $20 \%$, ofrece un notable incremento de la sensibilidad, con una aceptable disminución de la especificidad (Cuadro 5). Estos hallazgos soportan la modificación del criterio de extravasación plasmática, sugerida por otros autores, con la que se podría identificar a más pacientes que requieren un mayor cuidado ${ }^{9}$.
La principal limitación de este estudio reside en que la información se obtuvo de forma retrospectiva y no fue posible incluir otras variables que podrían modificar el valor del hematocrito, como las transfusiones y el volumen de líquidos que se administraron. Por ello, sería recomendable validar los presentes resultados en estudios prospectivos que se deben diseñar para tal fin.

Cabe advertir que los días de evaluación intrahospitalaria no se van a correlacionar de modo perfecto con los días de evolución de los síntomas, debido a que los pacientes ingresaron en distintos momentos de la enfermedad. Por lo anterior, la Gráfica 1 no se debe interpretar como una descripción precisa de la historia natural del hematocrito en términos de otras variables, sino como una tendencia que podría explicar el grado de variabilidad observada en pacientes hospitalizados por dengue.

Otro aspecto importante consiste en que los individuos estudiados para ingresar en el análisis, debían contar por lo menos con dos hematocritos. En conse- 
cuencia, se deduce que los enfermos debían tener un seguimiento intrahospitalario suficiente para alcanzar este criterio y, quizá, un grado considerable de severidad para requerir ese control.

Por lo anterior, el estudio se basa en una población situada en el extremo más severo del espectro clínico del dengue. Por tanto, es de esperar que la variabilidad sea menor, lo que conduce a una reducción en la magnitud de las asociaciones. Sin embargo, esta característica del estudio no invalidaría sus hallazgos; al contrario, sugeriría que las asociaciones pueden ser más fuertes de lo evidenciado, en una población más heterogénea.

En resumen, los hallazgos expuestos sugieren que los pacientes con dengue severo que necesitan una mayor atención, por la presencia de trombocitopenia o hemorragias mayores, muestran una mayor variación del hematocrito. Estos resultados apoyan el uso de este parámetro en la toma de decisiones clínicas en personas con dengue. Asimismo, sugieren que un criterio más flexible, como el de un viraje del hematocrito mayor de $10 \%$ (en lugar de $20 \%$ ), podría ser útil y emplearse para definir la severidad del dengue.

Conflicto de intereses. Los autores declaran que no hay conflicto de intereses en el presente manuscrito.

\section{REFERENCIAS}

1. Kautner I, Robinson MJ, Kuhnle U. Dengue virus infection: Epidemiology, pathogenesis, clinical presentation, diagnosis, and prevention. J Pediatr. 1997; 131: 516-24.

2. Guzmán MG, Kouri GP, Bravo J, Soler M, Vázquez S, Morier L. Dengue hemorrhagic fever in Cuba, 1981: A retrospective seroepidemiologic study. Am J Trop Med Hyg. 1990; 42: 17984.

3. Pan American Health Organization. Dengue and dengue hemorrhagic fever in the Americas: guidelines for prevention and control. Sci Publ. 1994; 548: 69-70.

4. Camacho T, de la Hoz F, Cárdenas V, Sánchez C, de Calderón L, Pérez L, Bermúdez A. Incomplete surveillance of a dengue-
2 epidemic in Ibagué, Colombia, 1995-1997. Biomedica. 2004; 24: $174-82$.

5. Murgue B, Deparis X, Chungue E, Cassar O, Roche C. Dengue: an evaluation of dengue severity in French Polynesia based on an analysis of 403 laboratory-confirmed cases. Trop Med Int Health. 1999; 4: 765-73.

6. DíazFA, Díaz RG, Villar LA, Martínez RA. Aplicación de los criterios de dengue hemorrágico en pacientes hospitalizados. Biomedica. 2005; 25 (Sup 1): 196.

7. Balmaseda A, Hammond SN, Pérez MA, Cuadra R, Solano S, Rocha J, et al. Assessment of the World Health Organization scheme for classification of dengue severity in Nicaragua. $\mathrm{Am}$ J Trop Med Hyg. 2005; 73: 1059-62.

8. Díaz-Quijano FA, Martínez-Vega RA, Villar-Centeno LA. Indicators of severity in dengue. Enferm Infecc Microbiol Clin. 2005; 23: 529-32.

9. Rigau-Pérez JG, Bonilla GL. An evaluation of modified case definitions for the detection of dengue hemorrhagic fever. Puerto Rico Association of Epidemiologists. PR Health Sci J. 1999; 18: 347-52.

10. Wiwanitkit V, Manusvanich P. Can hematocrit and platelet determination on admission predict shock in hospitalized children with dengue hemorrhagic fever? A clinical observation from a small outbreak. Clin Appl Thromb Hemost. 2004; 10: 657.

11. Lum LC, Goh AY, Chan PW, El-Amin AL, Lam SK. Risk factors for hemorrhage in severe dengue infections. JPediatr. 2002; 140: 629-31.

12. Narayanan M, Aravind MA, Thilothammal N, Prema R, Sargunam CS, Ramamurty N. Dengue fever epidemic in Chennai: a study of clinical profile and outcome. Indian Pediatr. 2002; 39: 1027-33.

13. Gomber S, Ramachandran VG, Kumar S, Agarwal KN, Gupta P, Gupta P, Dewan DK. Hematological observations as diagnostic markers in dengue hemorrhagic fever: a reappraisal. Indian Pediatr. 2001; 38: 477-81.

14. Díaz-Quijano FA, Martínez-Vega RA, Ocazionez RE, VillarCenteno LA. Evaluación de la prueba de IgM en suero agudo para el diagnóstico del dengue en un área endémica. Enferm Infecc Microbiol Clin. 2006; 24: 90-2.

15. Saito M, Oishi K, Inoue S, Dimaano EM, Alera MT, Robles $\mathrm{AM}$, et al. Association of increased platelet-associated immunoglobulins with thrombocytopenia and the severity of disease in secondary dengue virus infections. Clin Exp Immunol. 2004; 138: 299-303. 